\title{
Inclusive dijet photoproduction in ultraperipheral heavy ion collisions at the CERN Large Hadron Collider in next-to-leading order QCD
}

\author{
V. Guzey ${ }^{1,2,3}$ and M. Klasen ${ }^{4}$ \\ ${ }^{1}$ Department of Physics, University of Jyväskylä, P.O. Box 35, FI-40014 Jyväskylä, Finland \\ ${ }^{2}$ Helsinki Institute of Physics, University of Helsinki, P.O. Box 64, FI-00014 Helsinki, Finland \\ ${ }^{3}$ National Research Center "Kurchatov Institute”, Petersburg Nuclear Physics Institute (PNPI), Gatchina 188300, Russia \\ ${ }^{4}$ Institut für Theoretische Physik, Westfälische Wilhelms-Universität Münster, Wilhelm-Klemm-Straße 9, 48149 Münster, Germany
}

(Received 7 March 2019; revised manuscript received 13 May 2019; published 25 June 2019)

\begin{abstract}
We compute the cross section of inclusive dijet photoproduction in ultraperipheral $\mathrm{Pb}-\mathrm{Pb}$ collisions at the CERN Large Hadron Collider using next-to-leading order perturbative QCD. We demonstrate that our theoretical calculations provide a good description of various kinematic distributions measured by the ATLAS Collaboration. We find that the calculated dijet photoproduction cross section is sensitive to nuclear modifications of parton distribution functions (PDFs) at the level of $10 \%$ to $20 \%$. Hence, this process can be used to reduce uncertainties in the determination of these nuclear PDFs, whose current magnitude is comparable to the size of the calculated nuclear modifications of the dijet photoproduction cross section.
\end{abstract}

DOI: 10.1103/PhysRevC.99.065202

\section{INTRODUCTION}

Ultraperipheral collisions (UPCs) of relativistic ions correspond to large impact parameters between the nuclei exceeding the sum of their radii, so that short-range strong interactions between the ions are suppressed and reactions proceed rather via the emission of quasireal photons by the colliding ions. Thus, UPCs allow one to study photon-photon and photon-hadron (proton, nucleus) interactions at high energies [1]. During the last decade, UPCs have become an active field of research, driven by experimental results obtained at the BNL Relativistic Heavy Ion Collider (RHIC) and the CERN Large Hadron Collider (LHC); for a recent experimental review see, e.g., [2]. Notable examples of various UPC processes and their analyses include the two-photon production of dilepton pairs [3,4]; light-by-light scattering $\gamma \gamma \rightarrow \gamma \gamma$ and searches for potential physics beyond the Standard Model [5-7]; an electromagnetic double-scattering contribution to dimuon pair production in photon-photon scattering [8]; exclusive photoproduction of charmonia in proton-proton $[9,10]$, proton-nucleus [11], and nucleus-nucleus [12-15] UPCs and of bottomonia in proton-proton [16] and proton-nucleus UPCs [17]; new constraints on the small- $x$ gluon distribution in the proton [18,19] and heavy nuclei $[20,21]$ and the dynamics of strong interactions at high energies in the color dipole framework [22-24]; and exclusive photoproduction of $\rho$ mesons on

Published by the American Physical Society under the terms of the Creative Commons Attribution 4.0 International license. Further distribution of this work must maintain attribution to the author(s) and the published article's title, journal citation, and DOI. Funded by $S C O A P^{3}$. nuclei [25-28] as well as tests of models of nuclear shadowing $[29,30]$.

Focusing on UPC studies of nuclear structure in QCD at the LHC, coherent $J / \psi$ photoproduction in Pb-Pb UPCs at $\sqrt{s_{N N}}=2.76 \mathrm{TeV}[12-15]$ revealed a significant nuclear suppression of the measured rapidity distributions. In the framework of the leading logarithmic approximation of perturbative QCD [31], it can be interpreted as evidence of large nuclear gluon shadowing, $R_{g}=f_{g / A}\left(x, \mu^{2}\right) /\left[A f_{g / N}\left(x, \mu^{2}\right)\right] \approx 0.6$ at $x=10^{-3}$ and $\mu^{2}=3 \mathrm{GeV}^{2}\left(f_{g / A}\right.$ and $f_{g / N}$ are gluon densities in $\mathrm{Pb}$ and the proton, respectively). This value of $R_{g}$ agrees with predictions of the leading twist nuclear shadowing model [32], which are characterized by small theoretical uncertainties in this kinematic region. It is also broadly consistent with the EPS09 [33], nCTEQ15 [34], and EPPS16 [35] nuclear parton distribution functions (nPDFs), which, however, have significant uncertainties in this kinematic regime. Note that in the collinear factorization framework, next-to-leading order (NLO) perturbative QCD corrections to the cross section of $J / \psi$ photoproduction are large [36,37] and the relation between the gluon parton distribution function (PDF) and the gluon generalized parton distribution (GPD) is model dependent, which makes it challenging to interpret the UPC data on $J / \psi$ photoproduction on nuclei in terms of the NLO gluon nPDF.

The program of UPC measurements continues with Run 2 at the LHC, where besides photoproduction of vector mesons, inclusive dijet photoproduction in $\mathrm{Pb}-\mathrm{Pb}$ UPCs $A A \rightarrow A+$ 2 jets $+X$ has also recently been measured by the ATLAS Collaboration [38] (for leading-order QCD predictions for rates of this process, see [39]). The cross section of this process is sensitive to quark and gluon nPDFs $f_{j / A}\left(x, \mu^{2}\right)$ in a wide range of the momentum fraction $x$ and the resolution scale $\mu>\mathcal{O}(20) \mathrm{GeV}$, where one still expects sizable nuclear modifications of the PDFs. In addition, imposing 


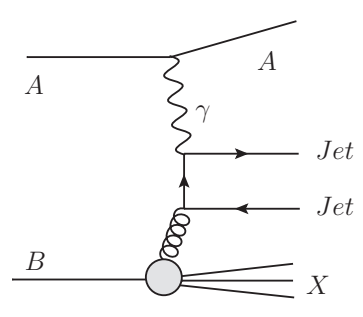

(a)

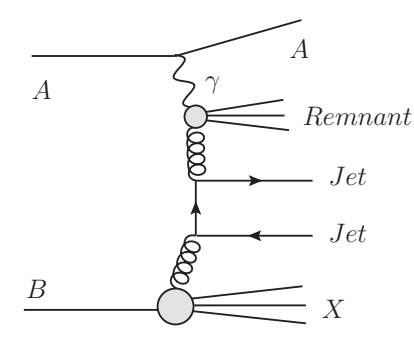

(b)
FIG. 1. Typical leading-order Feynman graphs for dijet photoproduction in UPCs of hadrons $A$ and $B$. Graphs (a) and (b) correspond to the direct and resolved photon contributions, respectively.

the requirement that the target nucleus stays intact, one can study diffractive dijet photoproduction in UPCs $A A \rightarrow A+$ 2 jets $+X+A$. Studies of this process may shed some light on the mechanism of QCD factorization breaking in diffractive photoproduction and, for the first time, give access to nuclear diffractive PDFs [40,41]. While further progress in constraining nPDFs will benefit from studies of high-energy hard processes with nuclei in proton-nucleus $(p A)$ scattering at the LHC [42] and lepton-nucleus $(e A)$ scattering at a future Electron-Ion Collider (EIC) [43] and Large Hadron Electron Collider (LHeC) [44], UPCs at the LHC present an important and complementary method of obtaining new constraints already now on $n P D F s$ in a wide kinematic range.

In this work, we make predictions for the cross section of inclusive dijet photoproduction in $\mathrm{Pb}-\mathrm{Pb}$ UPCs at the LHC using NLO perturbative QCD [45] and nCTEQ15 nPDFs. We show that our approach provides a good description of various cross section distributions measured by the ATLAS Collaboration [38]. Our analysis also shows that the dijet photoproduction cross section in the considered kinematics is sensitive to nuclear modifications of the PDFs. As a function of the momentum fraction $x_{A}$, the ratio of the cross sections calculated with nPDFs and in the impulse approximation behaves similarly to $R_{g}$ for a given $\mu$ and deviates from unity by $10-20 \%$ for the central nCTEQ15 fit. The calculations using EPPS16 nPDFs and predictions of the leading twist nuclear shadowing model give similar results. This suggests that inclusive dijet photoproduction on nuclei can be used to reduce uncertainties in the determination of nPDFs, which are currently significant and comparable in size to the magnitude of the calculated nuclear modifications of the dijet photoproduction cross section.

The remainder of this paper is structured as follows. In Sec. II, we outline the formalism of dijet photoproduction in UPCs using NLO perturbative QCD. We present and discuss our results for the $\mathrm{LHC}$ in Sec. III and draw conclusions in Sec. IV.

\section{PHOTOPRODUCTION OF DIJETS IN UPCS IN NLO PERTURBATIVE QCD}

Typical leading-order (LO) Feynman diagrams for dijet photoproduction in UPCs of nuclei $A$ and $B$ are shown in Fig. 1, where the graphs (a) and (b) correspond to the direct and resolved photon contributions, respectively. Note that beyond LO, the separation of the direct and resolved photon contributions depends on the factorization scheme and scale (see the discussion below).

Using the Weizsäcker-Williams method, which allows one to treat the electromagnetic field of an ultrarelativistic ion as a flux of equivalent quasireal photons $[1,46]$, and the collinear factorization framework for photon-nucleus scattering, the cross section of the UPC process $A B \rightarrow A+2$ jets $+X$ is given by [45]

$$
\begin{aligned}
d \sigma & (A B \rightarrow A+2 \text { jets }+X) \\
= & \sum_{a, b} \int_{y_{\min }}^{y_{\max }} d y \int_{0}^{1} d x_{\gamma} \int_{x_{A, \min }}^{x_{A, \max }} d x_{A} f_{\gamma / A}(y) f_{a / \gamma}\left(x_{\gamma}, \mu^{2}\right) f_{b / B} \\
& \times\left(x_{A}, \mu^{2}\right) d \hat{\sigma}(a b \rightarrow \text { jets }),
\end{aligned}
$$

where $a, b$ are parton flavors; $f_{\gamma / A}(y)$ is the flux of equivalent photons emitted by ion $A$, which depends on the photon light-cone momentum fraction $y ; f_{a / \gamma}\left(x_{\gamma}, \mu^{2}\right)$ is the PDF of the photon, which depends on the momentum fraction $x_{\gamma}$ and the factorization scale $\mu ; f_{b / B}\left(x_{A}, \mu^{2}\right)$ is the nuclear PDF with $x_{A}$ being the corresponding parton momentum fraction; and $d \hat{\sigma}(a b \rightarrow$ jets $)$ is the elementary cross section for production of two- and three-parton final states emerging as jets in hard scattering of partons $a$ and $b$. The sum over $a$ involves quarks and gluons for the resolved photon contribution and the photon for the direct photon contribution dominating at $x_{\gamma} \approx 1$. At LO, the direct photon contribution has support exactly only at $x_{\gamma}=1$, i.e., $f_{a / \gamma}=\delta\left(1-x_{\gamma}\right)$. At NLO, the virtual and real corrections are calculated with massless quarks in dimensional regularization, ultraviolet (UV) divergences are renormalized in the $\overline{\mathrm{MS}}$ scheme, and infrared (IR) divergences are canceled and factorized into the proton and photon PDFs, respectively. For the latter, this implies a transformation from the $\mathrm{DIS}_{\gamma}$ into the $\overline{\mathrm{MS}}$ scheme. The integration limits are determined by the rapidities and transverse momenta of the produced jets; see Sec. III. Note that Eq. (1) is based on the clear separation of scales, which characterize the longdistance electromagnetic interaction and the short-distance strong interaction. It generalizes the NLO perturbative QCD formalism of collinear factorization for jet photoproduction in lepton-proton scattering developed in Refs. [45,47-49], which successfully described HERA ep data on dijet photoproduction [50]. Hence, Eq. (1) involves universal nuclear PDFs $f_{b / B}\left(x_{A}, \mu^{2}\right)$, which can be accessed in a variety of hard processes involving nuclear targets [33-35], and the universal photon PDFs $f_{a / \gamma}\left(x_{\gamma}, \mu^{2}\right)$, which are determined by $e^{+} e^{-}$ data; for a review, see [45]. Hence, the interplay between the direct and resolved photon contributions in Eq. (1) is also universal and controlled by the standard $\mu^{2}$ evolution equations of photon PDFs and the choice of the factorization scheme.

In our analysis, we used the following input for Eq. (1). For photon PDFs $f_{a / \gamma}\left(x_{\gamma}, \mu^{2}\right)$, we used the GRV HO parametrization [51], which we transformed from the DIS ${ }_{\gamma}$ to the $\overline{\mathrm{MS}}$ factorization scheme. These photon PDFs have been profoundly tested at HERA and the Large Electron-Positron (LEP) collider at CERN and are very robust, in particular at high $x_{\gamma}$ (dominated by the pQCD photon-quark splitting), which is 
correlated with the low- $x_{A}$ gluons and sea quarks in $\mathrm{Pb}$ that present one of the points of interest of the present study. For nuclear PDFs $f_{b / B}\left(x_{A}, \mu^{2}\right)$, we employed the nCTEQ15 parametrization [34]. The photon flux $f_{\gamma / A}(y)$ produced by a relativistic point-like charge $Z$ is given by the standard expression

$$
f_{\gamma / A}(y)=\frac{2 \alpha_{\text {e.m. }} Z^{2}}{\pi} \frac{1}{y}\left[\zeta K_{0}(\zeta) K_{1}(\zeta)-\frac{\zeta^{2}}{2}\left[K_{1}^{2}(\zeta)-K_{0}^{2}(\zeta)\right]\right]
$$

where $\alpha_{\text {e.m. }}$ is the fine-structure constant; $K_{0,1}$ are modified Bessel functions of the second kind; $\zeta=y m_{p} b_{\min }$ with $m_{p}$ being the proton mass and $b_{\min }$ the minimal distance between two nuclei. For Pb-Pb UPCs, Eq. (2) with $b_{\min }=14.2 \mathrm{fm}$ reproduces very well the photon flux calculated taking into account the nuclear form factor and the suppression of strong interactions at impact parameters $b<b_{\min }$; see the discussion in [52].
The NLO calculation of the dijet photoproduction cross section using Eq. (1) is numerically implemented in an NLO parton-level Monte Carlo generator [45,47-49], which has been successfully tested in many different environments (HERA, LEP, Tevatron). It implements the anti- $k_{T}$ algorithm (but we have at most two partons in the jet) and all the kinematic conditions and cuts used in the ATLAS analysis [38] that are explicitly explained in the following section. Hadronization corrections and underlying event (UE) subtractions are not part of our analysis, but they are expected to be performed with PYTHIA simulations by the experiment once the data are final (as has been done at HERA).

\section{PREDICTIONS FOR DIJET PHOTOPRODUCTION IN Pb-Pb COLLISIONS AT THE LHC}

The main goal of the present paper is the first NLO QCD calculation of the cross section of inclusive dijet photoproduction in $\mathrm{Pb}-\mathrm{Pb}$ UPCs and concluding whether it can describe

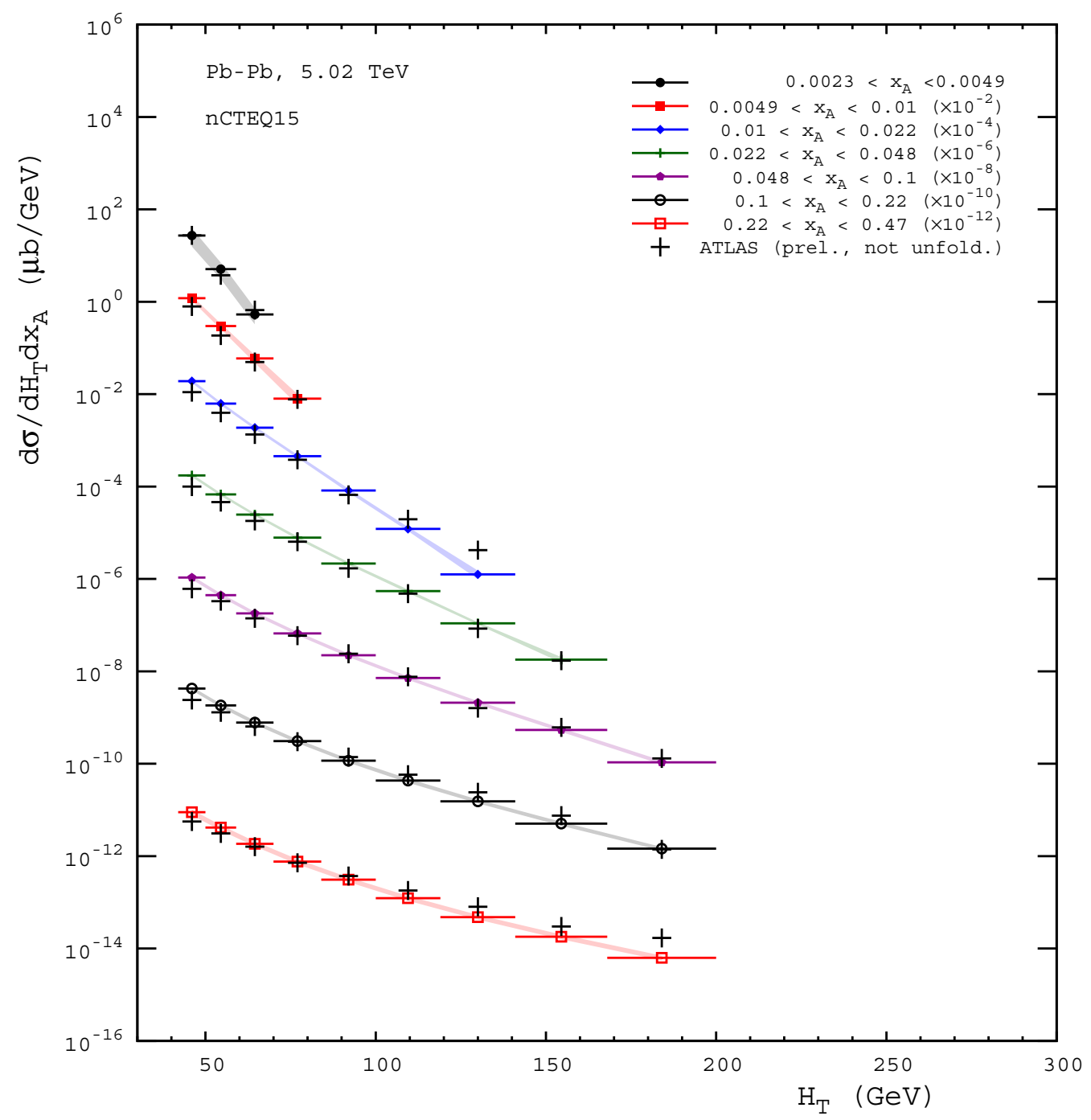

FIG. 2. NLO QCD predictions for the cross section of dijet photoproduction in Pb-Pb UPCs at $\sqrt{s_{N N}}=5.02 \mathrm{TeV}$ in the ATLAS kinematics as a function of $H_{T}$ for different bins of $x_{A}$. The central values and the corresponding shaded uncertainty bands are obtained using nCTEQ15 nPDFs. The crosses are the ATLAS data points that we extracted from [38]. 
the results of the ATLAS measurement [38]. The ATLAS analysis was performed using the following conditions and selection criteria:

(1) the anti- $k_{T}$ algorithm with the jet radius $R=0.4$;

(2) the leading jet has $p_{T, 1}>20 \mathrm{GeV}$, while the other jets have a different cut on $p_{T, i \neq 1}>15 \mathrm{GeV}$ as required [53], which corresponds to $35<H_{T}<400 \mathrm{GeV}$, where $H_{T}=\sum_{i} p_{T, i}$;

(3) all jets have rapidities $\left|\eta_{i}\right|<4.4$;

(4) the combined mass of all reconstructed jets is $35<$ $m_{\text {jets }}<400 \mathrm{GeV}$

(5) the parton momentum fraction on the photon side $z_{\gamma}=$ $y x_{\gamma}, 10^{-4}<z_{\gamma}<0.05$

(6) the parton momentum fraction on the nucleus side $x_{A}$, $5 \times 10^{-4}<x_{A}<1$.

The ATLAS results are presented as distributions in terms of the total jet transverse momentum $H_{T}=\sum_{i} p_{T, i}$ and the photon $z_{\gamma}$ and nucleus $x_{A}$ light-cone momentum fractions

$$
z_{\gamma}=\frac{m_{\mathrm{jets}}}{\sqrt{s_{N N}}} e^{y_{\mathrm{jets}}}, \quad x_{A}=\frac{m_{\mathrm{jets}}}{\sqrt{s_{N N}}} e^{-y_{\mathrm{jets}}},
$$

where

$$
\begin{aligned}
m_{\text {jets }} & =\left[\left(\sum_{i} E_{i}\right)^{2}-\left|\sum_{i} \vec{p}_{i}\right|^{2}\right]^{1 / 2}, \\
y_{\text {jets }} & =\frac{1}{2} \ln \left(\frac{\sum_{i} E_{i}+p_{z, i}}{\sum_{i} E_{i}-p_{z, i}}\right) .
\end{aligned}
$$

In Eqs. (4), the index $i$ runs over all measured jets; $E_{i}$ and $\vec{p}_{i}$ denote the jet energy and momentum, respectively. Note that, at LO, the kinematics of $2 \rightarrow 2$ parton scattering and the momentum fractions $z_{\gamma}$ and $x_{A}$ can be exactly reconstructed from the dijet measurement. At NLO, Eqs. (3) serve as hadron-level estimators of the momentum fractions entering Eq. (1); for brevity, we use the same notations in Eqs. (1) and (4).

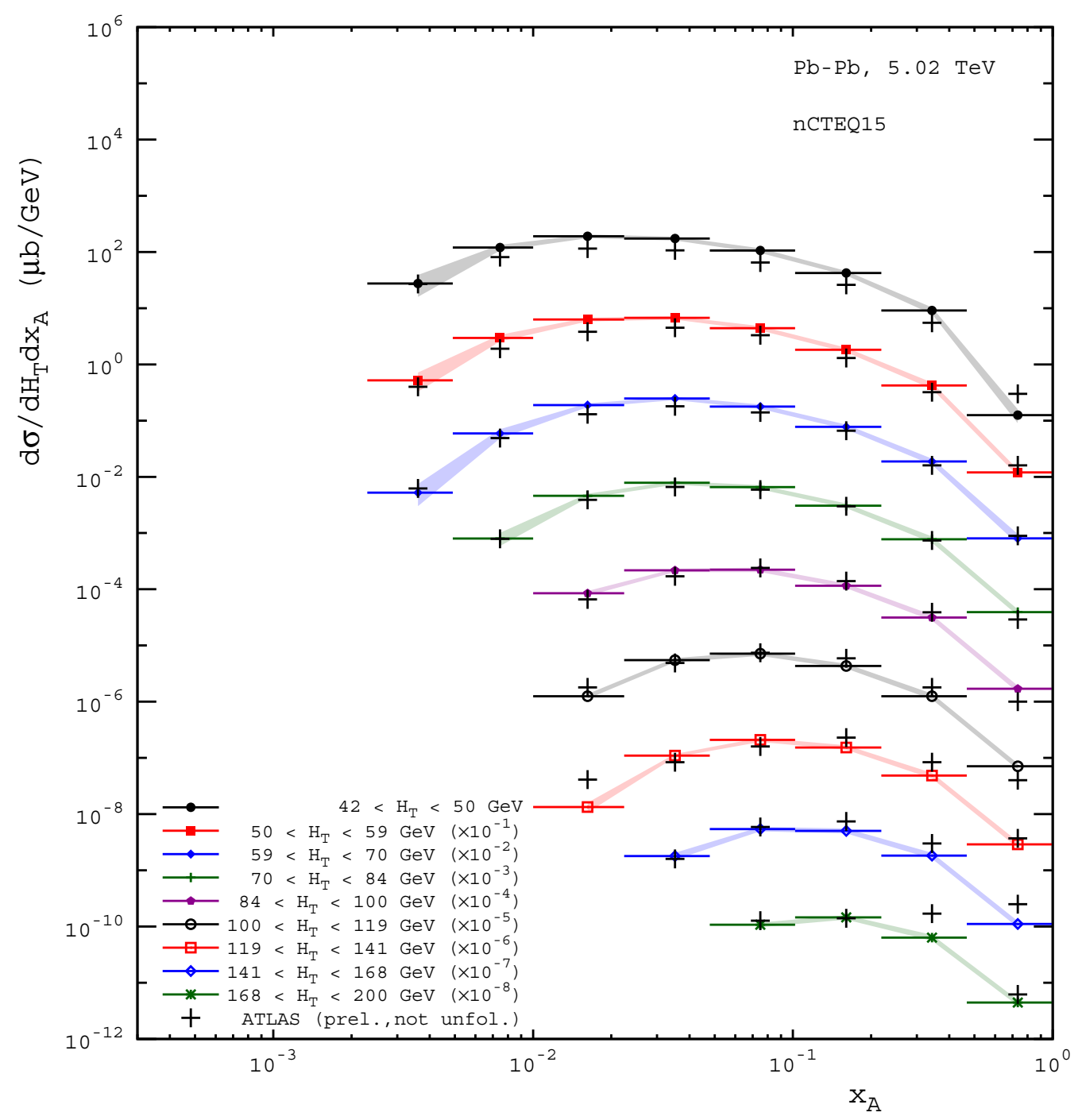

FIG. 3. NLO QCD predictions for the cross section of dijet photoproduction in Pb-Pb UPCs at $\sqrt{s_{N N}}=5.02$ TeV in the ATLAS kinematics as a function of $x_{A}$ for different bins of $H_{T}$. The crosses are the ATLAS data points that we extracted from [38]. 


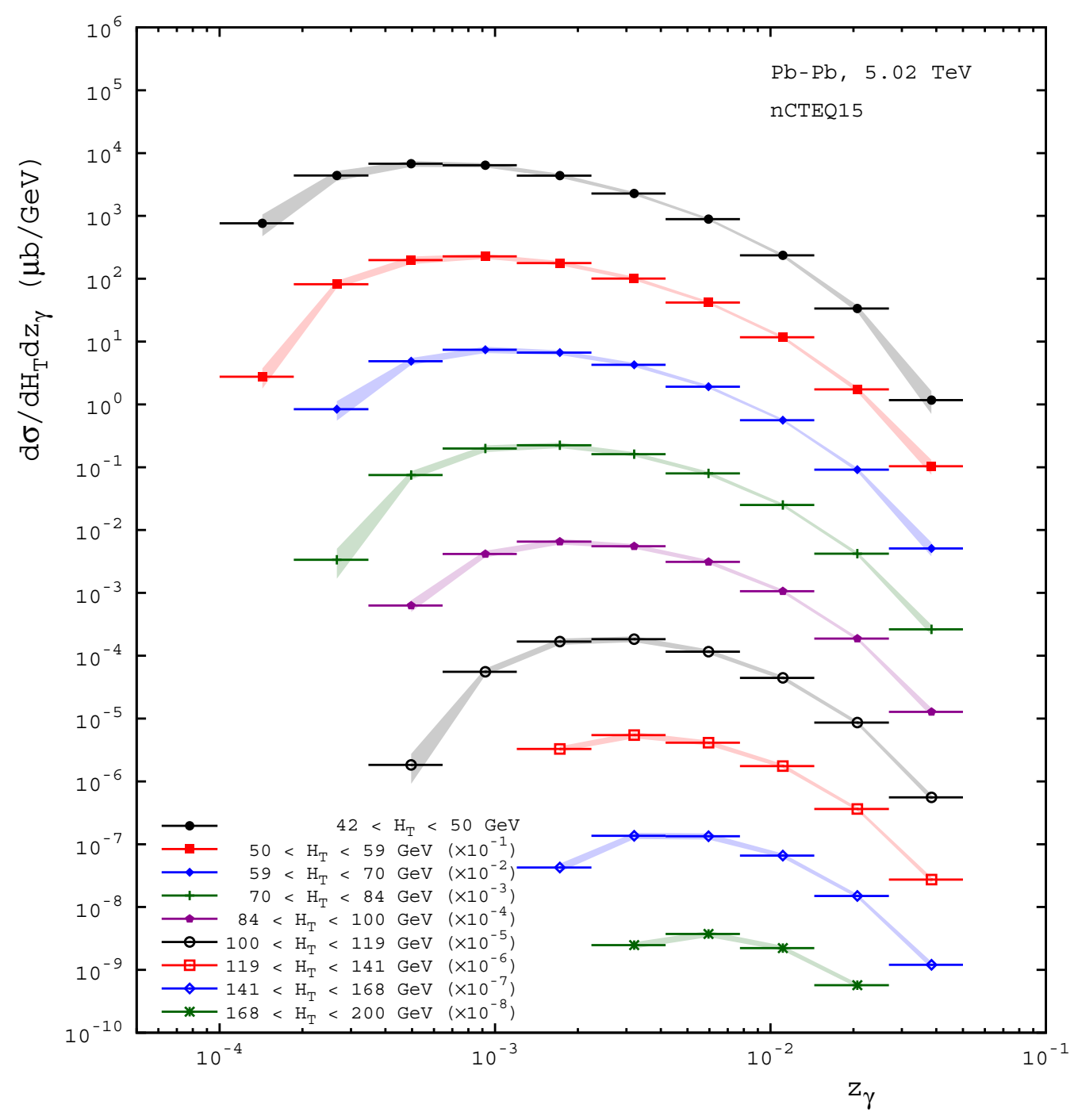

FIG. 4. NLO QCD predictions for the cross section of dijet photoproduction in Pb-Pb UPCs at $\sqrt{s_{N N}}=5.02$ TeV in the ATLAS kinematics as a function of $z_{\gamma}$ for different bins of $H_{T}$.

Figures 2, 3, 4, and 5 show our results for the cross section of dijet photoproduction in Pb-Pb UPCs in the ATLAS kinematics (see above) as a function of $H_{T}, x_{A}$, and $z_{\gamma}$ for different bins of these variables. They correspond to Figs. 12-15 of Ref. [38]. In each bin, our predictions are obtained using the central fit of nCTEQ15 nPDFs [34]. The shaded bands quantify the uncertainty of our results $\Delta \sigma$ due to the uncertainty of nCTEQ15 nPDFs. It is calculated by adding in quadrature the individual uncertainties corresponding to each of 32 error sets:

$$
\Delta \sigma=\frac{1}{2} \sqrt{\sum_{k=1, \text { odd }}^{31}\left[\sigma\left(f_{k}\right)-\sigma\left(f_{k+1}\right)\right]^{2}},
$$

where $\sigma\left(f_{k}\right)$ is the cross section calculated using the $f_{k}$ nCTEQ15 error nPDFs.

A comparison of our results shown in Figs. 2, 3, 4, and 5 with Figs. 12-15 of Ref. [38] demonstrates that our calculations describe well the corresponding distributions. To illustrate this point, in Figs. 2 and 3, which present the phenomenologically important distributions in $H_{T}$ and $x_{A}$, respectively, we also explicitly show by crosses the ATLAS data points, which we extracted from [38] using the WeBPLOTDIGITIZER tool [54]. One can readily see from these figures that the results of our calculations describe both the shape and normalization of the data rather well. The description of the remaining two distributions is also adequate. Note that the ATLAS data are preliminary and have not been corrected (unfolded) for the detector response.

To assess the impact of measurements of the inclusive dijet photoproduction cross section on nPDFs, we focus on the $x_{A}$ distribution. Our results are shown in Figs. 6 and 7. In Fig. 6, we show the ratio of the cross section calculated using nCTEQ15 nPDFs in lead to the one calculated in the impulse approximation (IA), where nuclear PDFs are assumed not to include any nuclear modifications and are given by the weighted sum of free proton and neutron PDFs, $f_{b / A}^{\mathrm{IA}}=$ $Z f_{b / p}+(A-Z) f_{b / n}$. The panels in this figure corresponds to nine bins in $H_{T}$ presented in Fig. 3 (the numerator of the presented ratio is given by the curves in Fig. 3). One can see that 


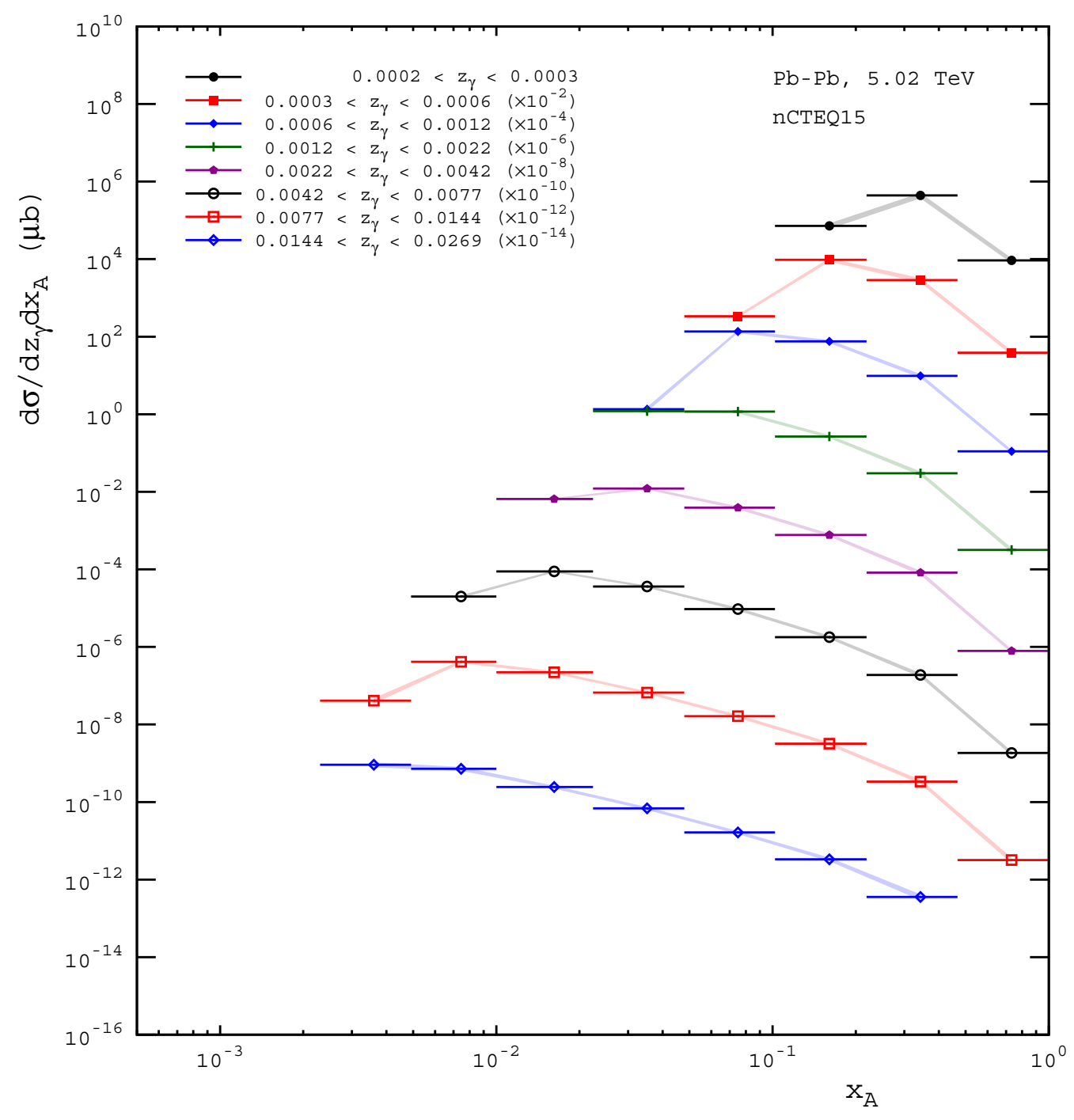

FIG. 5. NLO QCD predictions for the cross section of dijet photoproduction in Pb-Pb UPCs at $\sqrt{s_{N N}}=5.02$ TeV in the ATLAS kinematics as a function of $x_{A}$ for different bins of $z_{\gamma}$.

the cross section ratio as a function of $x_{A}$ behaves similarly to the ratio $R_{g}=f_{g / A}\left(x, \mu^{2}\right) /\left[A f_{g / N}\left(x, \mu^{2}\right)\right]$ of the nuclear and nucleon gluon distributions. It dips below unity for $x_{A}<$ 0.01 due to nuclear shadowing and then becomes enhanced around $x_{A}=0.1$ due to the assumed gluon antishadowing. For $x_{A}>0.3$, the cross section ratio shows again a suppression due to the EMC effect encoded in the nPDFs. While the scaling violations (the $H_{T}$ dependence) of the shown ratios are difficult to see, they seem to be positive at small $x \sim 0.01$ and negative at large $x$, as they should be; cf. [34,35].

Note that in spite of large values of the resolution scale probed in the considered kinematics, $\mu>\mathcal{O}(20) \mathrm{GeV}$, one can see that results are still sensitive to nuclear modifications of the PDFs at the 10-20\% level for the central value of our predictions. One should also note that the uncertainty due to nPDFs, which is given by the shaded bands, is significant and comparable to the size of the discussed nuclear modifications. This can be viewed as an opportunity to reduce uncertainties of nPDFs using data on cross section of inclusive dijet photoproduction in nuclei in global QCD fits of nPDFs.
In Fig. 7, we show our predictions for the dijet cross section as a function of $x_{A}$ integrated over $H_{T}$ and $z_{\gamma}$. The top panel presents separately the resolved (green, dot-dashed) and the direct (blue, dashed) photon contributions to the cross section as well as their sum (red, solid). As can be expected, because of the correlation between $x_{A}$ and $z_{\gamma}$ [see Eq. (3)], the resolved photon contribution dominates for $x_{A}>0.01$. We find that for small $x_{A}<0.01$ the two contributions are comparable, with the direct contribution being somewhat larger. While this behavior is qualitatively similar to the results of the LO analysis in the framework of PYTHIA 8 with EPPS16 nPDFs [55], the relative contribution of the resolved photon term is larger at NLO, but this statement depends of course on the choice of the photon factorization scheme and scale.

The middle panel of Fig. 7 presents the ratio to the impulse approximation. Similarly to the trend already observed in Fig. 6 and discussed above, this ratio as a function of $x_{A}$ behaves similarly to $R_{g}$. This behavior is similar to the one observed in the case of dijet photoproduction in the kinematics of an Electron-Ion Collider (EIC) [56]. 


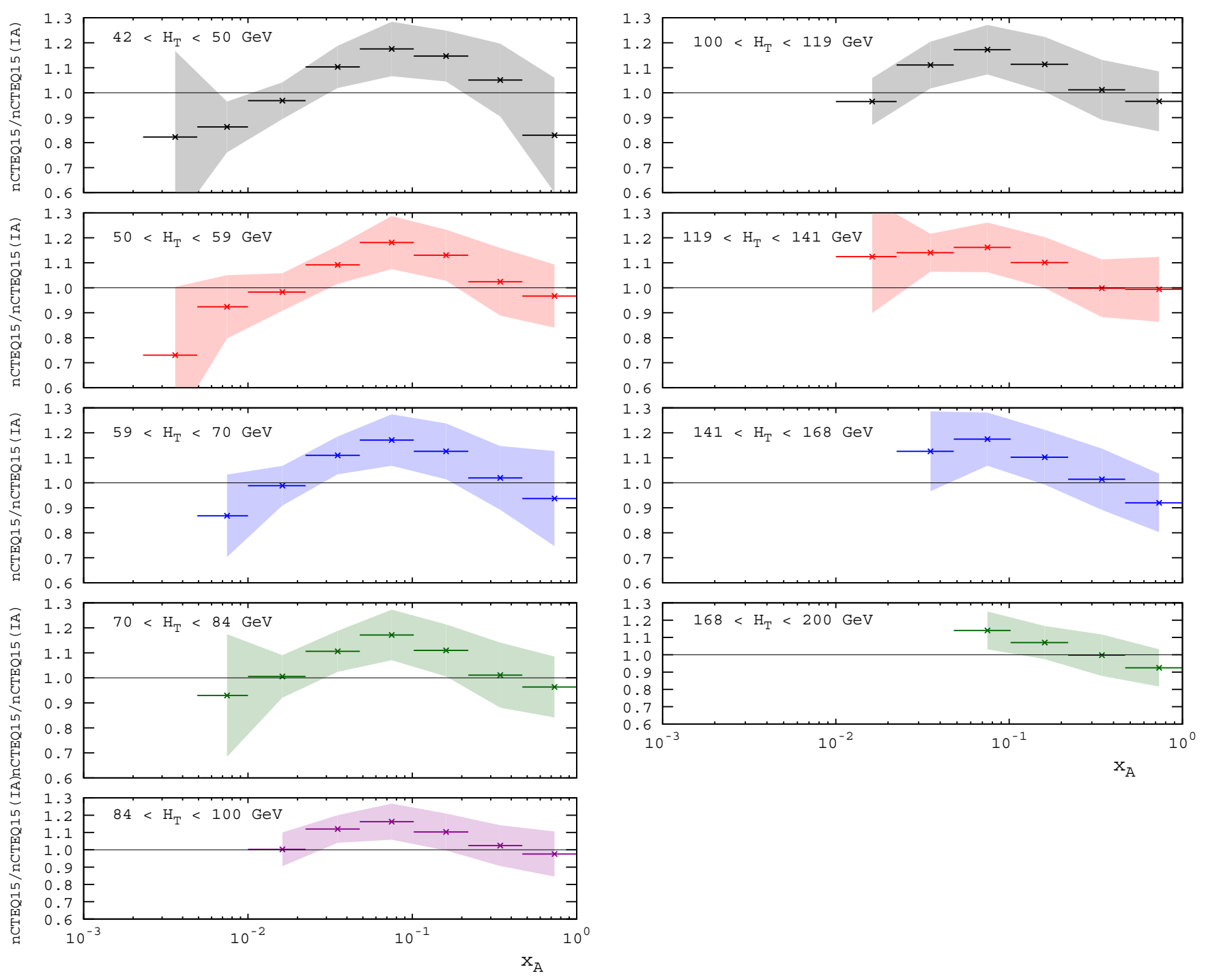

FIG. 6. NLO QCD predictions for the ratio of the cross section of dijet photoproduction to that calculated in IA in Pb-Pb UPCs at $\sqrt{s_{N N}}=$ $5.02 \mathrm{TeV}$ in the ATLAS kinematics as a function of $x_{A}$ for different $H_{T}$ bins. The shaded bands give the uncertainty of nCTEQ15 nPDFs.

Note that in the future and if/when experimental data become available, to study nuclear modifications of nPDFs one can directly form the ratio of dijet cross sections measured in $\mathrm{Pb}-\mathrm{Pb}$ and proton-proton $(p p)$ UPCs as a function of $x_{A}$. While the systematics are highly correlated between bins in $x_{A}$, the information on $\mathrm{nPDFs}$ is in the shape of the cross section ratio, see the middle panel of Fig. 7 and its discussion above. Also, while the central $\mathrm{Pb}-\mathrm{Pb}$ and $p p$ collisions are very different, $\mathrm{Pb}-\mathrm{Pb}$ and $p p$ UPCs have comparable multiplicities. Thus, one can expect that the systematic uncertainties largely cancel in the nucleus-to-proton cross section ratio.

Finally, the bottom panel of Fig. 7 presents the ratio of the dijet cross section calculated using nCTEQ15 nPDFs to the one calculated with the central value of EPPS16 nPDFs. The shaded band quantifies the uncertainty of the nCTEQ15 fit. One can see from the panel that the two parametrizations of nPDFs give similar predictions, which differ by at most $5 \%$ for all but one values of $x_{A}$. We have also explicitly checked that the use of nPDFs calculated in the model of leading twist nuclear shadowing [32] gives similarly close predictions for the dijet photoproduction cross section.

In our calculations, following the standard prescription for setting the hard scale in QCD calculations, we used $\mu=2 E_{T, 1}$ in Eq. (1). In detail, we performed calculations using $\mu=$ $\left(E_{T, 1} / 4, E_{T, 1} / 2, E_{T, 1}, 2 E_{T, 1}, 4 E_{T, 1}\right)$ both at NLO and LO and found that (i) the integrated cross section of inclusive dijet photoproduction at NLO as a function of $\mu$ is approximately constant is the vicinity of $\mu=2 E_{T, 1}$, (ii) while the NLO cross section slightly increases with an increase of $\mu$ up to $2 E_{T, 1}$ and then starts to decrease again, the LO cross section steeply decreases monotonically, and (iii) the values of the two cross sections are close around $\mu=2 E_{T, 1}$. Therefore, $\mu=2 E_{T, 1}$ in Eq. (1) corresponds to the choice, which is most numerically stable against higher-order corrections.

In this work, we used the framework of collinear factorization and NLO perturbative QCD to examine the sensitivity of the dijet photoproduction cross section to nuclear modifications of PDFs. Alternatively, one can use this process 

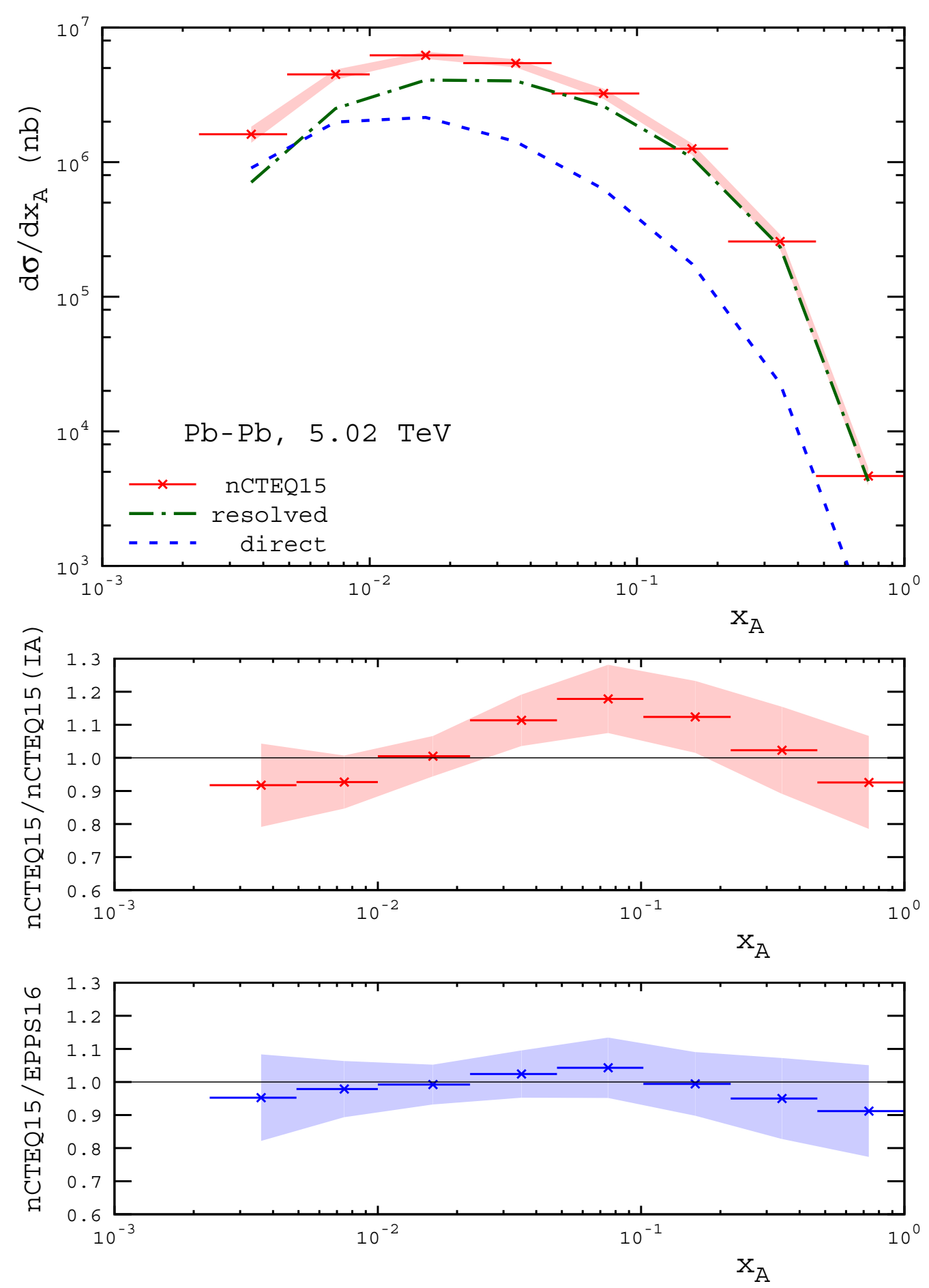

FIG. 7. NLO QCD predictions for the cross section of dijet photoproduction in Pb-Pb UPCs at $\sqrt{s_{N N}}=5.02$ TeV in the ATLAS kinematics as a function of $x_{A}$. Top: The resolved (green, dot-dashed) and direct (blue, dashed) photon contributions and their sum (red, solid). Middle: The ratio to the impulse approximation. Bottom: The ratio of cross sections calculated using the nCTEQ15 and EPPS16 nPDFs. The shaded bands show the uncertainty of nCTEQ15 nPDFs.

to look for signs of the Balitsky-Fadin-Kuraev-Lipatov and gluon saturation dynamics in the high-energy $\left(k_{T}\right)$ factorization approach [57].

\section{CONCLUSIONS}

In this work, we calculated the cross section of inclusive dijet photoproduction in $\mathrm{Pb}-\mathrm{Pb}$ UPCs at the LHC using NLO 
perturbative QCD and nCTEQ15 nPDFs. We showed that our approach provides a good description of various cross section distributions measured by the ATLAS Collaboration. We found that the calculated dijet photoproduction cross section is sensitive to nuclear modifications of the PDFs. In particular, as a function of the nucleus momentum fraction $x_{A}$, the ratio of the cross sections calculated with nPDFs and in the impulse approximation behaves similarly to $R_{g}$ for given $\mu$ and deviates from unity by $10-20 \%$ for the central nCTEQ15 fit. The calculations using EPPS16 nPDFs and predictions of the leading twist nuclear shadowing model give similar results. Therefore, inclusive dijet photoproduction on nuclei has the potential to reduce uncertainties in determination of nPDFs, which are comparable to the magnitude of the calculated nuclear modifications of the dijet photoproduction cross section. Our present analysis is a step in this direction.

\section{ACKNOWLEDGMENTS}

V.G. would like to thank A. Angerami, I. Helenius, K. Kovarik, H. Paukkunen, and M. Strikman for useful discussions and the Institut für Theoretische Physik, Westfälische Wilhelms-Universität Münster for hospitality. V.G.'s research is supported in part by RFBR, research Project No. 17-5212070. The authors gratefully acknowledge financial support of DFG through the Grant No. KL 1266/9-1 within the framework of the joint German-Russian project "New constraints on nuclear parton distribution functions at small $x$ from dijet production in $\gamma A$ collisions at the LHC."
[1] A. J. Baltz et al., Phys. Rep. 458, 1 (2008).

[2] S. R. Klein, Nucl. Phys. A 967, 249 (2017).

[3] S. R. Klein, Phys. Rev. C 97, 054903 (2018).

[4] V. P. Gonçalves, M. M. Jaime, D. E. Martins, and M. S. Rangel, Phys. Rev. D 97, 074024 (2018).

[5] D. d'Enterria and G. G. da Silveira, Phys. Rev. Lett. 111, 080405 (2013); 116, 129901(E) (2016).

[6] S. Fichet, G. von Gersdorff, B. Lenzi, C. Royon, and M. Saimpert, J. High Energy Phys. 02 (2015) 165.

[7] M. Kłusek-Gawenda, P. Lebiedowicz, and A. Szczurek, Phys. Rev. C 93, 044907 (2016).

[8] A. van Hameren, M. Kłusek-Gawenda, and A. Szczurek, Phys. Lett. B 776, 84 (2018).

[9] R. Aaij et al. (LHCb Collaboration), J. Phys. G 40, 045001 (2013).

[10] R. Aaij et al. (LHCb Collaboration), J. Phys. G 41, 055002 (2014).

[11] B. B. Abelev et al. (ALICE Collaboration), Phys. Rev. Lett. 113, 232504 (2014).

[12] E. Abbas et al. (ALICE Collaboration), Eur. Phys. J. C 73, 2617 (2013).

[13] B. Abelev et al. (ALICE Collaboration), Phys. Lett. B 718, 1273 (2013).

[14] J. Adam et al. (ALICE Collaboration), Phys. Lett. B 751, 358 (2015).

[15] V. Khachatryan et al. (CMS Collaboration), Phys. Lett. B 772, 489 (2017).

[16] R. Aaij et al. (LHCb Collaboration), J. High Energy Phys. 09 (2015) 084.

[17] R. Chudasama et al. (CMS Collaboration), PoS ICPAQGP 2015, 042 (2017), arXiv:1607.00786.

[18] S. P. Jones, A. D. Martin, M. G. Ryskin, and T. Teubner, J. High Energy Phys. 11 (2013) 085.

[19] V. Guzey and M. Zhalov, J. High Energy Phys. 10 (2013) 207.

[20] A. Adeluyi and C. A. Bertulani, Phys. Rev. C 85, 044904 (2012).

[21] V. Guzey, E. Kryshen, M. Strikman, and M. Zhalov, Phys. Lett. B 726, 290 (2013).

[22] T. Lappi and H. Mäntysaari, Phys. Rev. C 87, 032201(R) (2013).

[23] V. P. Goncalves, B. D. Moreira, and F. S. Navarra, Phys. Rev. C 90, 015203 (2014).
[24] H. Mäntysaari and B. Schenke, Phys. Lett. B 772, 832 (2017).

[25] C. Adler et al. (STAR Collaboration), Phys. Rev. Lett. 89, 272302 (2002).

[26] B. I. Abelev et al. (STAR Collaboration), Phys. Rev. C 77, 034910 (2008).

[27] G. Agakishiev et al. (STAR Collaboration), Phys. Rev. C 85, 014910 (2012).

[28] J. Adam et al. (ALICE Collaboration), J. High Energy Phys. 09 (2015) 095.

[29] V. Rebyakova, M. Strikman, and M. Zhalov, Phys. Lett. B 710, 647 (2012).

[30] L. Frankfurt, V. Guzey, M. Strikman, and M. Zhalov, Phys. Lett. B 752, 51 (2016).

[31] M. G. Ryskin, Z. Phys. C 57, 89 (1993).

[32] L. Frankfurt, V. Guzey, and M. Strikman, Phys. Rep. 512, 255 (2012).

[33] K. J. Eskola, H. Paukkunen, and C. A. Salgado, J. High Energy Phys. 04 (2009) 065.

[34] K. Kovarik et al., Phys. Rev. D 93, 085037 (2016).

[35] K. J. Eskola, P. Paakkinen, H. Paukkunen, and C. A. Salgado, Eur. Phys. J. C 77, 163 (2017).

[36] D. Y. Ivanov, A. Schäfer, L. Szymanowski, and G. Krasnikov, Eur. Phys. J. C 34, 297 (2004); 75, 75(E) (2015).

[37] S. P. Jones, A. D. Martin, M. G. Ryskin, and T. Teubner, J. Phys. G 43, 035002 (2016).

[38] The ATLAS Collaboration, Photo-nuclear dijet production in ultra-peripheral $\mathrm{Pb}+\mathrm{Pb}$ collisions, CERN Report No. ATLASCONF-2017-011, February 2017 (unpublished).

[39] M. Strikman, R. Vogt, and S. N. White, Phys. Rev. Lett. 96, 082001 (2006).

[40] V. Guzey and M. Klasen, J. High Energy Phys. 04 (2016) 158.

[41] E. Basso, V. P. Goncalves, A. K. Kohara, and M. S. Rangel, Eur. Phys. J. C 77, 600 (2017).

[42] C. A. Salgado et al., J. Phys. G 39, 015010 (2012).

[43] A. Accardi et al., Eur. Phys. J. A 52, 268 (2016).

[44] J. L. Abelleira Fernandez et al. (LHeC Study Group), J. Phys. G 39, 075001 (2012).

[45] M. Klasen, Rev. Mod. Phys. 74, 1221 (2002).

[46] C. A. Bertulani, S. R. Klein, and J. Nystrand, Annu. Rev. Nucl. Part. Sci. 55, 271 (2005).

[47] M. Klasen and G. Kramer, Z. Phys. C 72, 107 (1996). 
[48] M. Klasen and G. Kramer, Z. Phys. C 76, 67 (1997).

[49] M. Klasen, T. Kleinwort, and G. Kramer, Eur. Phys. J. direct C 1, 1 (1998), arXiv:hep-ph/9712256.

[50] M. Klasen and G. Kramer, Eur. Phys. J. C 71, 1774 (2011).

[51] M. Glück, E. Reya, and A. Vogt, Phys. Rev. D 46, 1973 (1992).

[52] J. Nystrand, Nucl. Phys. A 752, 470 (2005).
[53] M. Klasen and G. Kramer, Phys. Lett. B 366, 385 (1996).

[54] http://arohatgi.info/WebPlotDigitizer.

[55] I. Helenius, PoS DIS 2018, 113 (2018).

[56] M. Klasen and K. Kovarik, Phys. Rev. D 97, 114013 (2018).

[57] P. Kotko, K. Kutak, S. Sapeta, A. M. Stasto, and M. Strikman, Eur. Phys. J. C 77, 353 (2017). 